\title{
Implicit self-esteem in borderline personality and depersonalization disorder
}

\author{
Alexis N. Hedrick ${ }^{1 \dagger}$ and Heather A. Berlin ${ }^{2 *}$ \\ ${ }^{1}$ Department of Psychology, Vassar College, Poughkeepsie, NY, USA \\ 2 Department of Psychiatry, Mount Sinai School of Medicine, New York, NY, USA
}

Edited by:

Hugo Critchley, University of Sussex, UK

\section{Reviewed by:}

Hugo Critchley, University of Sussex, UK

Nick Medford, Brighton and Sussex Medical School, UK

\section{*Correspondence:}

Heather A. Berlin, Department of Psychiatry, Mount Sinai School of Medicine, One Gustave L. Levy Place, Box 1230, New York, NY 10029, USA. e-mail: heather.berlin@mssm.edu

\section{${ }^{\dagger}$ Present address:}

Alexis N. Hedrick, Child Psychiatry Branch, National Institute of Mental Health, Bethesda, MD, USA.
Self-perception is disrupted in people with borderline personality disorder (BPD) and depersonalization disorder (DPD), fluctuating with sudden shifts in affect in BPD and experienced as detached in DPD. Measures of implicit self-esteem (ISE), free from conscious control and presentation biases, may highlight how such disruptions of self-concept differentially affect these two populations on an unconscious level. We examined ISE using the Implicit Association Test, along with measures of emotion, behavior, and temperament, in BPD $(n=18)$, DPD $(n=18)$, and healthy control $(n=35)$ participants. DPD participants had significantly higher ISE and were more harm avoidant than BPD and control participants, while BPD participants had more "frontal" behaviors and impulsivity and less self-directedness and cooperativeness than DPD and control participants. Thus, while BPD and DPD commonly overlap in terms of dissociative symptoms and emotional irregularities, differences in self-esteem, behavior, and temperament can help identify where they diverge in terms of their cognition, behavior, and ultimately underlying neurobiology.

Keywords: implicit self-esteem, borderline personality disorder, depersonalization disorder, dissociation

\section{INTRODUCTION}

Sense of self is disrupted for individuals with borderline personality disorder (BPD) and with depersonalization disorder (DPD). In $\mathrm{BPD}$, this global feeling of self-identity and worth is one of several unstable attributes, which include unstable "interpersonal relationships, self-image, and affects, and marked impulsivity" (American Psychiatric Association, 2000, p. 706). Their self-image - as well as affect, cognition, and behavior - fluctuates with their perception of rejection or abandonment (American Psychiatric Association, 2000). These abandonment fears arise from unstable interpersonal relationships where they initially idealize another person, then criticize, and devalue them. This reflects a defensive mechanism called "splitting" where representations of the self and others are perceived as all good or all bad, and instead of successfully integrating these representations, they oscillate between the two with shifts in their affect (Lynum et al., 2008; Myers and Zeigler-Hill, 2008). DPD, in contrast, is characterized by "a persistent or recurrent feeling of being detached from one's mental processes or body that is accompanied by intact reality testing," in the DSM-IV-TR (American Psychiatric Association, 2000, p. 519). Individuals with DPD may feel like they are automatons, as if they are living in a movie or dream, or may feel outside of their thoughts or body, but they are aware that it is just a feeling (American Psychiatric Association, 2000). Given that aspects of self-representation, such as self-worth and self-concept, are disrupted in each of these disorders, we investigated how implicit self-esteem (ISE) is affected in these populations.

Depersonalization is thought to be the result of a psychological defense mechanism where negative thoughts about oneself or events of one's life are split off, such that they are experienced as disconnected and therefore not traumatic (Guralnik et al., 2000). Specific cognitive deficits in DPD patients, including difficulties on measures of attention, short-term memory, and spatial reasoning, may also underlie subjective experiences of perceptual disturbances (Guralnik et al., 2000). Evidence suggests that people with DPD are in a heightened state of alert, yet their response to emotional stimuli is attenuated (Phillips et al., 2001; Sierra et al., 2002). Sierra and Berrios (1998) posit a corticolimbic disconnection model of DPD whereby hyperactivity of the right prefrontal cortex [particularly the right dorsolateral prefrontal cortex (DLPFC)] increases alertness, while left prefrontal activation inhibits the amygdala and other limbic structures, causing hypoemotionality. Findings from a PET study by Simeon et al. (2000) suggest that a disconnection may occur earlier in the emotional processing stream; they found functional differences only in sensory associative cortical regions, with no metabolic differences in prefrontal or anterior cingulate regions. However, later functional neuroimaging studies of DPD lend some support to the theory that dampened subjective experience of emotion is due to increased activation of frontal regions with concomitant decreased activation in areas of emotional perception such as anterior cingulate, insula, and amygdala (Phillips et al., 2001; Medford et al., 2006). While the neurobiological underpinnings are still not understood, specific perceptual, cognitive, and affective deficits in DPD are well known.

Patients with BPD have also been found to have cognitive deficits in a wide range of domains, but with significant impairment in executive functions like planning, cognitive flexibility, and response inhibition, which suggests a deficit in orbitofrontal cortex (OFC) (Berlin and Rolls, 2004; Berlin et al., 2005). Furthermore, 
neuroimaging studies have found structural and functional abnormalities of the OFC in BPD (e.g., Soloff et al., 2000; Tebartz van Elst et al., 2003). Given its role in inhibition, dysfunction of the OFC in BPD patients may underlie their failure to inhibit perceived negative emotional information or erroneous interpretations, potentially contributing to dysregulated self-esteem.

Borderline personality disorder and DPD are classified as distinct disorders by the DSM-IV-TR (American Psychiatric Association, 2000), however they commonly overlap in their symptomology. In particular, there is a high incidence of dissociative symptoms in BPD, as well as high comorbidity of BPD and dissociative disorders, especially DPD (Sar et al., 2006; Zanarini and Jager-Hyman, 2009). Studies of unconscious personality organization, such as ISE - which arises from automatic associations with the self and unconscious processing of affective experiences, as opposed to the deliberate, conscious self-evaluation of explicit self-esteem - may highlight differences in self-perception in these two disorders.

Despite problems with sense of self in these disorders, there is a paucity of research examining self-esteem in people with BPD and DPD. Self-esteem refers to overall feelings of self-worth (Buhrmester et al., 2011), and is not generally considered to be relative, or based on, valuation of things or other people. To our knowledge, no studies have been conducted on self-esteem in DPD to date. A study by Michal et al. (2006) found that patients with DPD report feeling extremely helpless, fragile, worthless, and socially isolated, which may contribute to, or emanate from lower self-esteem, but this was assessed with an inventory of narcissism rather than a measure of self-esteem. Others have described "an exaggerated and often unpleasant hyperawareness of the self" in DPD (Dell, 2009, p. 779), yet it remains unknown how this affects self-esteem.

More research has been done on self-esteem in BPD, demonstrating that like mood and evaluations of others, self-esteem may fluctuate between extreme highs and lows. Several studies have found a correlation between BPD features (BPDF) and labile, low self-esteem (Watson, 1998; Tolpin et al., 2004; Zeigler-Hill and Abraham, 2006). Further, Lynum et al. (2008) found that participants with BPD had higher explicit self-esteem as reported on the Self-Esteem Index than those with avoidant personality disorder (APD), although both groups scored in the clinical range of low self-esteem. They hypothesize that higher self-esteem in BPD is similarly due to fluctuations in mood and identity in this population, which contrast consistently low self-esteem in APD. While these studies provide evidence for an association between low, fluctuating self-esteem, and borderline personality characteristics, they all used subjective self-report measures of explicit self-esteem. Because explicit self-esteem is subject to conscious reflection, these results could be influenced by participants' presentation biases and limited capability of introspection and thus affected by BPD patients' volatile emotions. Instead, implicit measures can be employed in order to avoid capturing self-presentation.

Research supports the idea that people have an implicit as well as explicit attitude toward themselves (Greenwald and Banaji, 1995). Explicit self-esteem arises from reflective, conscious evaluation of self-relevant information, while ISE is automatic, intuitive, and unconscious (Greenwald and Banaji, 1995; Schröder-Abé et al., 2007). Thus explicit self-esteem can be assessed with direct measures such as questionnaires, but ISE requires indirect measures that assess one's reaction to self-relevant stimuli. While people are able to explicitly report on their self-concept with some accuracy, research shows that this is subject to influence by demand characteristics such as social desirability, and may not even be fully available to us given limitations in introspection (Greenwald and Banaji, 1995). Furthermore, these two constructs are considered independent; implicit and explicit self-esteem are weakly correlated, and can even be at odds with one another (Greenwald and Farnham, 2000; Vater et al., 2010). Typical, healthy adults tend to demonstrate congruent self-esteem, with a positive bias on both implicit and explicit self-esteem (Valiente et al., 2011). Discrepancies between the two (high explicit/low implicit, high implicit/low explicit) are relatively common, however, and indicate inner emotional turmoil or maladjustment (Schröder-Abé et al., 2007). In BPD, Vater et al. (2010) found that having either kind of selfesteem discrepancy is positively correlated with BPD symptom severity.

In sum, self-concept appears to be disrupted in people with BPD and DPD, but in different ways, fluctuating with sudden shifts in affect in BPD and experienced as detached in DPD. Measures of self-esteem may highlight how such disruptions of the concept of self differentially affect these two populations. Implicit measures in particular may better capture underlying differences in self-esteem that are free from conscious control and presentation biases that can influence explicit measures. Thus, we examined self-esteem in DPD and BPD in this study using Greenwald and Farnham (2000) adapted Implicit Association Test (IAT), which captures self-esteem based on one's ability to evaluate good and bad concepts as self-associated or other-associated. We hypothesized that the two patient groups would respond differently from one another, and that people with DPD and BPD would show lower self-esteem than controls on this implicit measure. We also administered measures of impulsivity, executive function, and affect to further explore the relationship between self-esteem and the symptoms of these psychiatric disorders.

\section{MATERIALS AND METHODS SUBJECTS}

A total of 71 adults participated in the study. The BPD and DPD participants were outpatients, diagnosed by an independent psychiatrist using the Structured Clinical Interview for DSM-IV Axis I Disorders (First et al., 2002), the Structured Clinical Interview for DSM-IV Axis II Disorders (First et al., 1997), the Structured Clinical Interview for DSM-IV Dissociative Disorders (Steinberg, 1994), and the Wechsler Abbreviated Scales of Intelligence (WASI; Wechsler, 1999) Patients were included for the study if they met DSM-IV-TR criteria for BPD $(N=18)$ or DPD $(N=18)$, and if they had no history of schizophrenia, schizoaffective, bipolar, or other organic mental disorders, no diagnosis of substance dependence or abuse, and no unstable medical disorder. Presence of a dissociative disorder was exclusionary for BPD participants, and vice versa. Healthy controls (HC; $N=35$ ) where recruited from the community using flyers and advertisements and were only included if they met no Axis I or II DSM-IV-TR criteria and had no neurological or unstable medical disorder. Participants where matched for age, gender, and IQ (as measured by the WASI; see Table 1). This study was approved by the institutional review board 
Table 1 | Demographics.

\begin{tabular}{lcccc}
\hline Group & $\boldsymbol{N}$ & Gender, f:m & Age, mean (SD, range) & I.Q. (WASI total), mean (SD, range) \\
\hline BPD & 18 & $12: 6$ & $33.00(9.505,21-51)$ & $106.4(18.753,73-142)$ \\
DPD & 18 & $9: 9$ & $34.67(10.387,23-55)$ & $117.19(17.406,97-165)$ \\
HC & 35 & $16: 19$ & $34.66(11.956,20-63)$ & $108.22(15.231,83-135)$
\end{tabular}

$f: m$, Female: male ratio; SD, standard deviation.

at Mount Sinai School of Medicine and all subjects signed written informed consent prior to participation.

\section{MATERIALS AND PROCEDURES Implicit association task}

A measure of ISE designed to test the strength of implicit associations between categories by measuring the speed of their simultaneous evaluation. Participants sat in a quiet room with no windows in front of a computer screen and were instructed by a female test administrator to sort the words presented in the center of the screen into either "self" or "other," or "pleasant" or "unpleasant" categories as quickly as possible by pressing the corresponding key on the left ("e") or right ("i") side of the keyboard. The category words were shown in the upper right and upper left sides of the screen and the target words were shown in the middle of the screen (see Table 2 for stimulus words). The IAT involved five steps, as described by Greenwald et al. (1998). In step one, a practice block of 24 trials, participants sorted self or other words, such as "me" and "their," by pressing the " $\mathrm{e}$ " and " $\mathrm{i}$ " keys respectively. In step two (24 practice trials), they sorted pleasant and unpleasant words, like "joy" and "pain," by pressing the "e" and "i" keys respectively. In step three (60 trials), participants were presented with self, other, pleasant, and unpleasant category words, categorizing both self and pleasant with the same key ("e"), and other and unpleasant with the other key ("i"). This was the congruent condition. In step four (24 trials), the keys corresponding to self and to other category words switched (now self was paired with the "i" key and other with the "e" key). In step five, the incongruent condition, participants categorized self and unpleasant words with the same key ("i") and other and pleasant words with the other key ("e"). The order of blocks was randomized for each participant. Stimulus words remained on the screen until the correct response was made, and the computer measured the reaction time between presentation of the stimulus word and the participants' correct categorization of the word.

The IAT is based on the assumption that categorizing a bipolar target concept (e.g., self or other) will be faster when it shares a stronger association with a bipolar attribute concept (e.g., pleasant or unpleasant) and when classifying these concepts requires the same response. It is expected that participants with high selfesteem will have faster response times when they must respond with the same button for the target ("self") and positive attribute ("pleasant") that are already implicitly associated, but that their reaction time will be slower when responding requires cognitive control to reverse this association, i.e., when "self" related words are paired with "unpleasant" negative attributes (Greenwald and Farnham, 2000). This assumption is supported by findings
Table 2 | IAT stimulus words.

\begin{tabular}{llll}
\hline Self & Other & Pleasant & Unpleasant \\
\hline Self & Them & Smile & Tragedy \\
My & It & Sunshine & Agony \\
Mine & Their & Rainbow & Grief \\
Me & They & Paradise & Death \\
I & Other & Warmth & Sickness \\
Myself & Themselves & Joy & Poison \\
& & Pleasure & Pain \\
& & Happy & Vomit \\
\hline
\end{tabular}

that healthy volunteers in fact demonstrate a self-positive bias (Greenwald and Farnham, 2000; Buhrmester et al., 2011).

Frontal behavior questionnaire (Berlin and Rolls, 2004; Berlin et al., 2005)

A self-report 20-item, 5-point Likert scale questionnaire that was designed to measure types of behavioral problems such as disinhibition, social inappropriateness, perseveration, and cooperativeness, generally believed to result from orbitofrontal damage (Levin et al., 1991).

\section{Subjective Emotion Questionnaire (Berlin and Rolls, 2004; Berlin et al., 2005)}

Measures how often participants experience sadness, anger, fear, happiness, and disgust in their current daily life on a 4-point Likert scale.

\section{Barratt Impulsiveness Scale-11 (Patton and Stanford, 1995)}

This 30-item, 4-point Likert scale self-report measure assesses long-term patterns of impulsive behavior. It asks subjects about the way they act and think without relation to any specific period of time, and it is used as a trait measure of impulsivity. The Barratt Impulsiveness Scale-11 (BIS-11) consists of three subscales: attentional/cognitive (i.e., rapid shifts and impatience with complexity), motor (i.e., acting without thinking), and non-planning (i.e., lack of future orientation).

\section{Temperament and Character Inventory (Cloninger et al., 1994), version 9}

This 240 item, true-false personality inventory includes the following temperament dimensions: novelty seeking (NS), a tendency toward exploratory activity, intense excitement in response to novelty, impulsive decision-making, and active avoidance of monotony or frustration; harm avoidance (HA), inhibition and 
social withdrawal, shyness, and slow adaptation to change; reward dependency (RD), a tendency to respond intensely to reward signals, particularly those of social approval; and Persistence $(\mathrm{P})$, resistance to extinction despite intermittent reinforcement. The questionnaire also measures three character dimensions: self-directedness, cooperativeness, and self-transcendence.

\section{STATISTICAL ANALYSES}

A one-way analysis of variance (ANOVA) was performed on each variable to determine whether mean scores differed significantly by group. While this resulted in a large number of independent ANOVAs, our sample size did not permit the use of a multivariate approach given the number of outcome measures of interest (Tabachnick and Fidell, 2007). Least Significant Difference (LSD) post hoc analyses were performed in order to identify the specific source of the difference and a false discovery rate test was run to correct for multiple comparisons. Effect size was calculated using Cohen's $d$. Although a multivariate regression was not appropriate given our sample size, Pearson's correlations were run within each group with ISE scores and each of the other measures that revealed significant results from the ANOVA. In Figure 1, error bars represent the SE, and $* p<0.0 .05, * * p<0.01$, and $* * * p<0.001$ with respect to control participants. An alpha level of 0.05 was used for all statistical procedures.

\section{DATA TRANSFORMATION AND ANALYSIS OF IAT}

Data from the two critical steps, (1) where participants categorized self with pleasant and other with unpleasant (congruent condition), and (2) where participants categorized self with unpleasant and other with pleasant (incongruent condition), were used for the primary analysis. Consistent with the data transformation presented by Greenwald et al. (1998), response latencies greater than

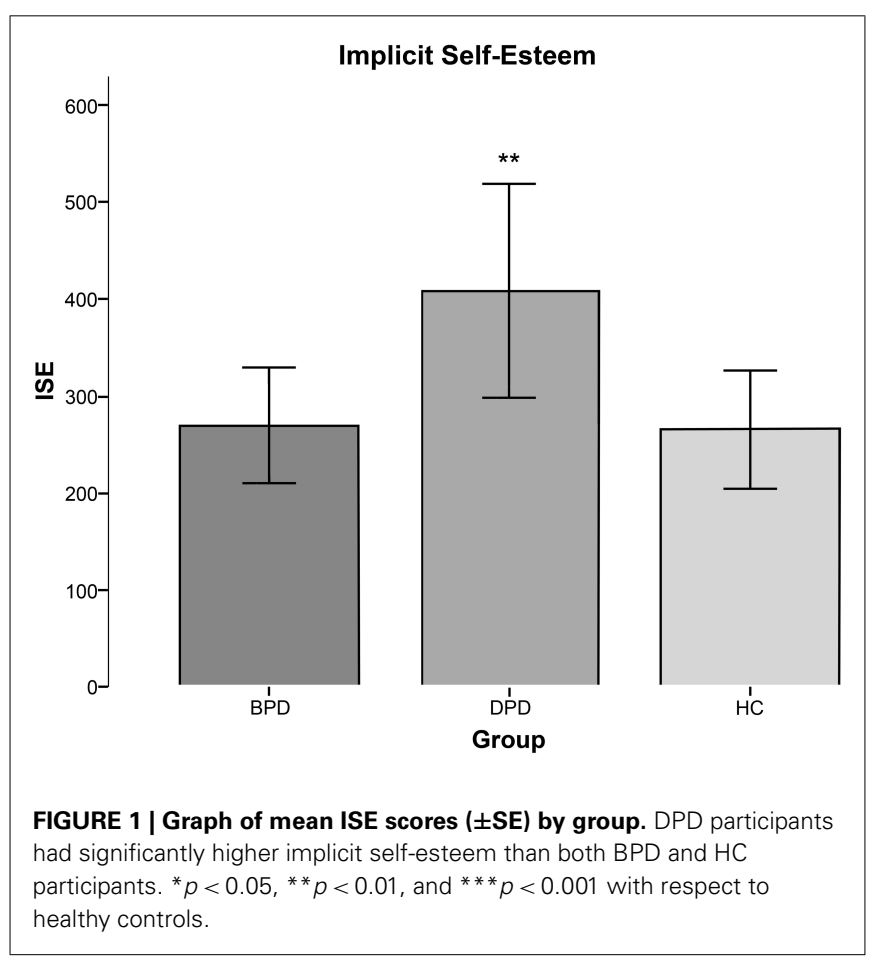

$3,000 \mathrm{~ms}$ were recoded to $3,000 \mathrm{~ms}$, and latencies less than $300 \mathrm{~ms}$ were recoded to $300 \mathrm{~ms}$ in order to eliminate the effect of extreme response latencies, where participants either hit the correct key before perceiving the stimulus or had a lapse in attention that caused these outliers. Additionally, participants who responded to $>10 \%$ of trials within the two critical blocks faster than $300 \mathrm{~ms}$ were to be removed, as recommended by Greenwald et al. (2003), however, no participants met this criterion. When participants made an error categorizing the stimulus word, reaction times were measured until they responded correctly, and these trials were included in the analysis (per Greenwald et al., 2003). The IAT effect - the measure of ISE - was then calculated by subtracting the mean latency of the self/pleasant reaction times (congruent trials) from that of the self/unpleasant blocks (incongruent trials). Thus a high score is indicative of high self-esteem.

\section{RESULTS}

A one-way ANOVA revealed no significant differences in age, gender, or IQ (as measured by the WASI) between groups (see Table 1), and showed that BPD and DPD participants differed significantly on ISE, frontal behaviors, impulsivity, and several temperament characteristics (see Table 3 ).

\section{IMPLICIT ASSOCIATION TASK}

Implicit self-esteem was calculated by subtracting the mean latency of the self/pleasant reaction times (congruent trials) from that of the self/unpleasant blocks (incongruent trials). A high score, therefore, is indicative of high self-esteem. We found that ISE was significantly related to group $[F(2,68)=4.02, p<0.05]$, and LSD post hoc analyses indicated that DPD participants had significantly higher ISE than HC participants $(p<0.01)$ and BPD participants $(p<0.05)$. Self-esteem was not significantly different between HC and BPD participants. Mean ISE scores by group are shown in Figure 1.

\section{FRONTAL BEHAVIOR QUESTIONNAIRE}

Frontal Behavior scores were significantly different between groups $[F(2,65)=17.488, p<0.001]$. Post hoc analyses found that BPD participants had significantly higher Frontal Behavior Questionnaire (FBQ) scores than both DPD participants $(p<0.01)$ and HCs $(p<0.001)$. Individuals with DPD also had significantly higher FBQ scores than HCs $(p<0.01)$.

\section{SUBJECTIVE EMOTION OUESTIONNAIRE}

Analyses of variance found significant group differences in scores for total subjective emotion $[F(2,66)=12.808, p<0.001]$, sadness $[F(2,66)=29.130, p<0.001]$, anger $[F(2,66)=12.709$, $p<0.001]$, fear $[F(2,66)=14.934,<0.001]$, and happiness $[F(2$, $66)=15.304, p<0.001]$. Disgust was the only emotion that did not show significant differences between groups. Post hoc analyses revealed that BPD and DPD participants similarly reported significantly higher scores than HCs for total subjective emotion $(p<0.001$ and 0.01 , respectively), sadness $(p<0.001$ for both $)$, anger (both $p<0.001$ ), and fear (both $p<0.001$ ), and significantly lower than HCs on the happiness subscale $(p<0.001$ for both groups). BPD and DPD participants did not significantly differ from one another on any subscale of the Subjective Emotion Questionnaire (SEQ). 
Table 3 | Means, SDs, and effect sizes for each self-report questionnaire, by group.

\begin{tabular}{|c|c|c|c|c|c|c|}
\hline & \multicolumn{3}{|c|}{ Mean score (SD) } & \multicolumn{3}{|c|}{ Effect size $(d)$} \\
\hline & BPD & DPD & $\mathrm{HC}$ & BPD vs HC & DPD vs HC & BPD vs DPD \\
\hline Subjective Emotion Questionnaire (total) & $7.12(1.54)^{* * *}$ & $6.44(2.83)^{* *}$ & $4.40(1.59)$ & -1.73 & -1.00 & 0.30 \\
\hline Sadness & $1.75(0.58)^{* * *}$ & $1.78(0.81)^{* * *}$ & $0.60(0.55)$ & -2.05 & -1.80 & 0.00 \\
\hline Anger & $1.44(0.63)^{* * *}$ & $1.28(0.83)^{* * *}$ & $0.60(0.50)$ & -1.55 & -1.10 & 0.22 \\
\hline Happiness & $1.44(0.63)^{* * *}$ & $1.28(0.67)^{* * *}$ & $2.20(0.63)$ & 1.20 & 1.43 & 0.25 \\
\hline Disgust & $1.00(0.82)$ & $0.72(0.83)$ & $0.51(0.61)$ & -0.72 & -0.30 & 0.34 \\
\hline Barratt Impulsivity Scale (total) & $73.33(11.20) * * *$ & $65.25(10.78) * * *$ & $49.81(9.14)$ & -2.38 & -1.60 & 0.74 \\
\hline Non-planning impulsivity & $28.05(5.38)^{* * *}$ & $24.89(3.96)^{* * *}$ & $19.95(4.57)$ & -1.66 & -1.10 & 0.67 \\
\hline Motor impulsivity & $25.19(4.34)^{* * *}$ & $21.83(5.04) * *$ & $18.54(3.29)$ & -1.80 & -0.80 & 0.71 \\
\hline Reward dependence & $15.47(3.46)$ & $16.81(4.36)$ & $15.93(4.31)$ & 0.11 & -0.20 & -0.30 \\
\hline Persistence & $4.40(1.96)$ & $5.19(2.11)$ & $5.37(1.94)$ & 0.50 & 0.09 & -0.40 \\
\hline Self-directedness & $20.47(5.41)^{* * *}$ & $26.88(7.17)^{* * *}$ & 38.37 (3.92) & 4.01 & 2.18 & -1.00 \\
\hline Cooperativeness & $28.60(7.07)^{* * *}$ & $32.69(7.04)$ & $35.93(4.59)$ & 1.33 & 0.58 & -0.60 \\
\hline Self-transcendence & $17.00(8.60)^{* *}$ & $13.56(8.17)$ & $10.67(5.59)$ & -0.90 & -0.40 & 0.41 \\
\hline
\end{tabular}

${ }^{*} p<0.05,{ }^{*} p<0.01$, and ${ }^{* *} p<0.001$ with respect to healthy controls. Items in bold indicate variables where BPD patients were also significantly different than DPD patients. Effect size was measured with Cohen's d.

\section{BARRATT IMPULSIVITY SCALE}

Total impulsivity scores $[F(2,66)=34.698, p<0.001]$, as well as attentional $[F(2,66)=43.493, p<0.001]$, motor $[F(2$, $66)=15.805, p<0.001]$, and non-planning impulsivity subscores $[F(2,66)=19.037, p<0.001]$ were significantly related to group. Post hoc analyses reveal that BPD participants had significantly higher total impulsivity scores compared to HCs $(p<0.001)$ and DPD participants $(p=0.05)$. DPD total BIS scores, though lower than for BPD subjects, were also higher as compared to HC $(p<0.001)$. BPD and DPD participants both had similarly higher attentional BIS scores than control participants $(p<0.001$ for both comparisons), with no significant difference between the two clinical groups. BPD and DPD participants scored significantly higher on the motor and non-planning subscales than controls (motor: $p<0.001$ and $p=0.01$, respectively, non-planning: $p<0.001$ for both comparisons), and BPD participants were significantly greater than those with DPD ( $p=0.05$ for motor and non-planning).

\section{TEMPERAMENT AND CHARACTER INVENTORY}

Harm avoidance $[F(2,58)=24.878, p<0.001]$, self-directedness $[F(2,58)=63.019, p<0.001]$, cooperativeness $[F(2,58)=7.708$, $p<0.05]$, and self-transcendence $[F(2,58)=4.022, p<0.05]$ varied significantly by group, while NS, social reward dependence, and persistence did not.

LSD post hoc analyses show that BPD and DPD participants scored significantly higher on HA than HCs $(p<0.001$ for both comparisons), and DPD participants scored significantly higher than BPD participants $(p=0.05)$. Furthermore, both clinical groups had lower self-directedness scores than controls $(p<0.001$ for each) with BPD participants scoring significantly lower than DPD participants $(p<0.01)$. BPD participants also scored significantly lower on cooperativeness than controls $(p<0.001)$, and higher on self-transcendence than HCs $(p<0.01)$. There were no significant differences between DPD and HC participants, nor DPD participants and BPD on cooperativeness and self-transcendence subscales.

\section{CORRELATIONS}

The only significant correlation with ISE was between ISE and cooperativeness in HCs $(r=0.372)$.

\section{DISCUSSION}

Our results show that patients with BPD and DPD differ significantly in ISE, suggesting that disturbed self-construct in these two disorders influences unconscious self-worth differently. Interestingly, DPD participants had significantly higher ISE than BPD participants and controls. This was somewhat surprising, given that participants with DPD have reported negative perceptions of themselves (Michal et al., 2006). However, that study used an explicit, self-report narcissism inventory without any direct measures of self-esteem, and indices of related psychological outcomes have been shown to weakly correlate with implicit methods (Buhrmester et al., 2011). Taken together with evidence of the corticolimbic disconnection model (Sierra and Berrios, 1998), our findings may reflect a protective mechanism of dissociation in DPD that dampens automatic negative associations with the self. 
If depersonalization indeed results from an adaptive, opposing reaction mechanism of prefrontal activity and inhibited limbic emotional processing, DPD participants may have higher ISE scores because intact attentional systems allow them to be alert and to respond quickly in general, while at the same time the emotional salience of negative associations with the self is dampened. Neuroimaging studies suggest that individuals with DPD may lack the normal neural distinction between emotional and neutral stimuli (Phillips et al., 2001; Medford et al., 2006), thus emotion-processing areas may be less active and less able to facilitate self-negative associations quickly. Furthermore, DPD patients' performance on neuropsychological tasks also supports this disconnection hypothesis and inflated ISE. While DPD patients perform poorly on attention, short-term memory, and spatial reasoning tasks (Guralnik et al., 2000), they perform better than controls on set shifting and divided attention tasks sensitive to DLPFC function (DePrince and Freyd, 1999). Thus, DLPFC hyperactivation - increasing attention and inhibiting limbic structures - could mediate DPD patients' ability to dissociate and explain their higher ISE.

Depersonalization disorder participants' higher ISE could also reflect an opposite, compensatory process of denial, whereby they manifest a strong unconscious bias toward aligning themselves with good attributes, in addition to dissociating negative attributes. The IAT is limited in that the components of the congruence effect cannot be separated and analyzed. It seems likely, however, that people with DPD are primarily slower to associate negative words with themselves relative to positive words given their characteristic down-regulation of implicit emotional reactivity (Sierra and Berrios, 1998; Phillips et al., 2001). Furthermore, Michal et al. (2006) found that DPD participants do not display narcissistic characteristics, which could otherwise confound ISE. Thus their higher self-esteem likely does not reflect defensive inflation of self-worth.

Despite blunted emotional responsivity being a key feature of DPD (Simeon et al., 2008), our findings on the SEQ show that inner experience of emotion is not down-regulated. Previous studies have shown that individuals with DPD have reduced affective, autonomic, and neural responses to externally presented stimuli such as emotional faces or scenes (Phillips et al., 2001; Sierra et al., 2002, 2006; Simeon et al., 2008). Yet DPD patients experience significant distress over their feelings numbness and unreality (Oyebode, 2008). They may have lowered physiological responding to external emotional stimuli, but our results demonstrate that they still experience negative emotions and can subjectively report them.

In contrast to individuals with DPD, those with BPD did not differ significantly from HC on ISE. This may be explained by BPD patients' characteristic oscillations of emotion, identity, and selfand other-evaluations (Tolpin et al., 2004; Lynum et al., 2008). Additionally, the IAT itself shows moderate test-retest reliability, with an average $r=0.56$ across nine studies (Nosek et al., 2007). Our participants completed the IAT only once, therefore if ISE fluctuates in BPD as explicit self-esteem does, this may not be captured by the IAT due to its limited sensitivity to measure such changes. To better measure self-esteem lability in people with BPD, future studies should administer the IAT at multiple time points.
Although BPD participants did not differ from HCs on ISE, they were distinguished by having more frontal behaviors (indicative of OFC dysfunction), greater impulsivity, and less self-directedness than both DPD participants and HCs. Impulsivity has long been identified as a characteristic symptom of BPD (Berlin and Rolls, 2004; Berlin et al., 2005). Low self-esteem may be a contributing factor to impulsivity in some disorders (e.g., pathological gambling, Burton et al., 2000), however, our findings do not support this association in people with BPD or DPD.

Despite common hypoemotionality in DPD and emotional lability in BPD, results for subjective emotions did not distinguish DPD from BPD. Both groups had similarly elevated levels of sadness, anger, and fear, and reported less happiness than controls. Temperament scales, however, did illustrate differences between the two disorders. DPD participants reported greater HA, which may reflect heightened alertness from increased DLPFC activity or which may be a learned response to trauma that commonly precipitates depersonalization. In contrast, BPD participants had significantly lower scores on self-directedness, consistent with their reliance on perceptions of others' feelings or opinions in determining their own personal image and goals. BPD participants also had lower cooperativeness, which is consistent with their characteristically dysfunctional interpersonal relationships.

We expected self-esteem to be significantly related to personality and temperament traits, yet cooperativeness was the only personality trait that significantly correlated with ISE scores. Similar to results on the SEQ, this could be due to the difference between implicit and explicit measures. The personality and temperament traits in this study were measured by explicit self-report questionnaires that may not correlate with ISE. Future studies should administer implicit measures of emotion and temperament along with the IAT for self-esteem to explore the relationship between ISE, emotion, and personality further.

A limitation of the current study is that we did not include an explicit measure of self-esteem for comparison. Discrepancies between implicit and explicit self-esteem may be most indicative of disordered self-perception (Schröder-Abé et al., 2007), thus future studies should include an explicit measure in conjunction with the IAT. Another limitation is that the study did not include severity scores for BPD and DPD patients, thus we are unable to examine whether our ISE findings are associated with degrees of illness. Interpretation of the results is also limited in that ISE is calculated in terms of appraisal of one dichotomous category relative to another. Strong pleasant-self associations may in fact reflect more negative associations of "other," as opposed to indicating high self-esteem (Karpinski, 2004). ISE can also be affected by preceding contexts that influence whether one adopts a normative perspective (i.e., categorizing target concepts based on what others find pleasant/unpleasant) or a personal perspective (i.e., what they themselves find pleasant/unpleasant) for completing the task (Han et al., 2010). Finally, we recognize that due to the relative small sample sizes in the current study, it is difficult to generalize our findings. Therefore we intend to conduct a follow-up study with a larger study size and to include severity measures to investigate the potential mediating effect of ISE on the intensity of depersonalization and related psychopathology, which may allow for a better interpretation of our current findings. 


\section{CONCLUSION}

While people with BPD and DPD overlap in terms of emotional irregularities and dissociative symptoms (at least in a subgroup of BPD patients), our findings suggest that differences in self-esteem, behavior, and temperament can help identify where they diverge in terms of cognition, behavior, and ultimately underlying neurobiology. Future research should examine BPD patients' ISE scores at multiple time points, along with explicit measures, to determine if ISE fluctuates like self-report measures suggest. The IAT should also be administered to patients with other dissociative disorders, such as dissociative identity disorder and dissociative amnesia, to further explore the relationship between a disordered sense of self and self-esteem. Finally, neuroimaging can be used to

\section{REFERENCES}

American Psychiatric Association. (2000). Diagnostic and Statistical Manual, Fourth Edition TR. Washington, DC: American Psychiatric Association.

Berlin, H. A., and Rolls, E. T. (2004). Time perception, impulsivity, emotionality, and personality in selfharming borderline personality disorder patients. J. Pers. Disord. 18, 358-378.

Berlin, H. A., Rolls, E. T., and Iversen, S. D. (2005). Borderline personality disorder, impulsivity, and the orbitofrontal cortex. Am. J. Psychiatry 162, 2360-2373.

Buhrmester, M. D., Blanton, H., and Swann, W. B. (2011). Implicit selfesteem: nature, measurement, and a new way forward. J. Pers. Soc. Psychol. 100, 365-385.

Burton, S., Netemeyer, R. G., and Andrews, J. C. (2000). Modeling potential psychological risk factors of pathological gambling. J. Appl. Soc. Psychol. 30, 2058-2078.

Cloninger, C. R., Przybeck, T. R., Svrakic, D. M., and Wetzel, R. D. (1994). The Temperament and Character Inventory (TCI): A Guide to its Development and Use. St. Louis, MO: Center for Psychobiology of Personality, Washington University.

Dell, P. F. (2009). "Understanding dissociation," in Dissociation and the Dissociative Disorders: DSM V and Beyond, eds P. F. Dell and J. A. O'Neil (New York: Routledge), 709-825.

DePrince, A. P., and Freyd, J. J. (1999). Dissociative tendencies, attention, and memory. Psychol. Sci. 10, 449-452.

First, M. B., Spitzer, R. L., Gibbon, M., and Williams, J. B. W. (1997). Structured Clinical Interview for DSMIV Personality Disorders, (SCID-II). Washington, DC: American Psychiatric Press, Inc.
First, M. B., Spitzer, R. L., Gibbon, M., and Williams, J. B. W. (2002). Structured Clinical Interview for DSM-IV Axis I Disorders, Research Version, Patient Edition (SCID-I/P). New York: Biometrics Research, New York State Psychiatric Institute.

Greenwald, A. G., and Banaji, M. R. (1995). Implicit social cognition: attitudes, self- esteem, and stereotypes. Psychol. Rev. 102, 4-27.

Greenwald, A. G., and Farnham, S. D. (2000). Using the implicit association test to measure self-esteem and self-concept. J. Pers. Soc. Psychol. 79, 1022-1038.

Greenwald, A. G., McGhee, D. E., and Schwartz, J. L. K. (1998). Measuring individual differences in implicit cognition: the implicit association test. J. Pers. Soc. Psychol. 74, 1464-1480.

Greenwald, A. G., Nosek, B. A., and Banaji, M. R. (2003). Understanding and using the implicit association test: I. An improved scoring algorithm. J. Pers. Soc. Psychol. 85, 197-216.

Guralnik, O., Schmeidler, J., and Simeon, D. (2000). Feeling unreal: cognitive processes in depersonalization. Am. J. Psychiatry 157, 103-109.

Han, H. A., Czellar, S., Olson, M. A., and Fazio, R. H. (2010). Malleability of attitudes or malleability of the IAT? J. Exp. Soc. Psychol. 46, 286-298.

Karpinski, A. (2004). Measuring selfesteem using the IAT: the role of the other. Pers. Soc. Psychol. Bull. 30, 22-34.

Levin, H. S., Goldstein, F. C., Williams, D. H., and Eisenberg, H. M. (1991). "The contribution of frontal lobe lesions to the neurobehavioral outcome of closed head injury," in Frontal Lobe Function and Dysfunction, eds H. S. Levin, H. M. Eisenberg, and L. B. Benton (Oxford: Oxford University Press), 318-337.

examine the neural basis of ISE and self-identity in these populations. Based on previous research (e.g., Berlin et al., 2005) and the findings of the current study, we suggest that OFC dysfunction may contribute to the impulsivity and emotional lability of BPD patients, while increased DLPFC activation might underlie the down-regulation of emotion and high self-esteem observed in patients with DPD.

\section{ACKNOWLEDGMENTS}

We would like to thank Jennifer Bartz, Ph.D. for providing the IAT task, Daphne Simeon, M.D. for subject recruitment, Holly Hamilton, B.A. for test administration, and Eric Hollander, M.D. for providing the lab facilitates.

Lynum, L. I., Wilberg, T., and Karterud, S. (2008). Self-esteem in patients with borderline and avoidant personality disorders. Scand. J. Psychol. 49, 469-477.

Medford, N., Brierley, B., Brammer, M., Bullmore, E. T., David, A. S., and Phillips, M. L. (2006). Emotional memory in depersonalization disorder: a functional MRI study. Psychiatry Res. 148, 93-102.

Michal, M., Kaufhold, J., Overbeck, G., and Grabhorn, R. (2006). Narcissistic regulation of the self and interpersonal problems in depersonalized patients. Psychopathology 39, 192-198.

Myers, E. M., and Zeigler-Hill, V. (2008). No shades of gray: splitting and selfesteem instability. Pers. Individ. Dif. 45, 139-145.

Nosek, B. A., Greenwald, A. G., and Banaji, M. R. (2007) "The implicit association test at age 7: a methodological and conceptual review," in Automatic Processes in Social Thinking and Behavior, ed. J. A. Bargh (New York: Psychology Press), 265-292.

Oyebode, F. (2008). Sims' Symptoms of the Mind. Edinburgh: Elsevier.

Patton, J. H., and Stanford, M. S. (1995). Barratt ES: factor structure of the Barratt Impulsiveness Scale. J. Clin. Psychol. 51, 768-774.

Phillips, M. L., Medford, N., Senior, C., Bullmore, E. T., Suckling, J., and Brammer, M. J., Andrew, C., Sierra, M., Williams, S. C., David, A. S. (2001). Depersonalization disorder: thinking without feeling. Psychiatry Res. 108, 145-160

Sar, V., Akyuz, G., Kugu, N., Ozturk, E., and Ertem-Vehid, H. (2006). Axis I dissociative disorder comorbidity in borderline personality disorder and reports of childhood trauma. J. Clin. Psychiatry 67 1583-1590.
Schröder-Abé, M., Rudolph, A., and Schütz, A. (2007). High implicit self-esteem is not necessarily advantageous: discrepancies between explicit and implicit self-esteem and their relationship with anger expression and psychological health. Eur. J. Pers. 21, 319-339.

Sierra, M., and Berrios, G. E. (1998). Depersonalization: neurobiological perspectives. Biol. Psychiatry 44, 898-908.

Sierra, M., Senior, C., Dalton, J., McDonough, M., Bond, A., Phillips, M. A., O'Dwyer, A. M., and David, A. S. (2002). Autonomic response in depersonalization disorder. Arch. Gen. Psychiatry 59, 833-838.

Sierra, M., Senior, C., Phillips, M. L., and David, A. S. (2006). Autonomic response in the perception of disgust and happiness in depersonalization disorder. Psychiatry Res. 145, 225-231.

Simeon, D., Guralnik, O., Hazlett, E. A., Spiegel-Cohen, J., Hollander, E., and Buchsbaum, M. S. (2000). Feeling unreal: a PET study of depersonalization disorder. Am. J. Psychiatry 157, 1782-1788.

Simeon, D., Kozin, D. S., Segal, K., Lerch, B., Dujour, R., and Giesbrecht, T. (2008). De-constructing depersonalization: further evidence for symptom clusters. Psychiatry Res. 157, 303-306.

Soloff, P. H., Meltzer, C. C., Greer, P. J., Constantine, D., and Kelly, T. M. (2000). A fenfluramine-activated FDG-PET study of borderline personality disorder. Biol. Psychiatry 47, 540-554.

Steinberg, M. (1994). Structured Clinical Interview for DSM-IV Dissociative Disorders-Revised (SCID-D-R), Washington, DC: American Psychiatric Press.

Tabachnick, B. G., and Fidell, L. S. (2007). Using Multivariate Statistics, 5th Edn. Boston: Pearson Education. 
Tebartz van Elst, L., Hesslinger, B., Thiel, T., Geiger, E., Haegele, K., and Lemieux, L., Lieb, K., Bohus, M., Hennig, J., and Ebert, D. (2003). Frontolimbic brain abnormalities in patients with borderline personality disorder: a volumetric magnetic resonance imaging study. Biol. Psychiatry 54, 163-171.

Tolpin, L. H., Gunthert, K. C., Cohen, L. H., and O'Neill, S. C. (2004). Borderline personality features and instability of daily negative affect and self-esteem. J. Pers. 72, 111-137.

Valiente, C., Cantero, D., Vázquez, C., Sanchez, A., Provencio, M., and Espinosa, R. (2011). Implicit and explicit self-esteem discrepancies in paranoia and depression. J. Abnorm. Psychol. 120, 691-699.

Vater, A., Schröder-Abé, M., Schütz, A., Lammers, C. H., and Roepke, S. (2010). Discrepancies between explicit and implicit self-esteem are linked to symptom severity in borderline personality disorder. $J$. Behav. Ther. Exp. Psychiatry 41, 357-364.

Watson, D. C. (1998). The relationship of self-esteem, locus of control, and dimensional models to personality disorders. J. Soc. Behav. Pers. 13, 399-420.

Wechsler, D. (1999). Wechsler Abbreviated Scale of Intelligence (WASI). San Antonio: The Psychological Corporation.
Zanarini, M. C., and Jager-Hyman, S. (2009). "Dissociation in borderline personality disorder," in Dissociation and the Dissociative Disorders: DSM $V$ and Beyond, eds P. F. Dell and J. A. O'Neil (New York: Routledge), 487-493.

Zeigler-Hill, V., and Abraham, J. (2006). Borderline personality features: instability of self-esteem and affect. J. Soc. Clin. Psychol. 25, 668-687.

Conflict of Interest Statement: The authors declare that the research was conducted in the absence of any commercial or financial relationships that could be construed as a potential conflict of interest.
Received: 02 November 2011; accepted: 12 March 2012; published online: 05 April 2012.

Citation: Hedrick $A N$ and Berlin $H A$ (2012) Implicit self-esteem in borderline personality and depersonalization disorder. Front. Psychology 3:91. doi: 10.3389/fpsyg.2012.00091

This article was submitted to Frontiers in Consciousness Research, a specialty of Frontiers in Psychology.

Copyright (c) 2012 Hedrick and Berlin. This is an open-access article distributed under the terms of the Creative Commons Attribution Non Commercial License, which permits non-commercial use, distribution, and reproduction in other forums, provided the original authors and source are credited. 